\title{
EchoGéo
}

$26 \mid 2013$

Varia

\section{De la religion dans les sciences sociales aux sciences sociales dans la religion : l'exemple chrétien}

Frédéric Dejean

\section{CpenEdition}

Journals

Édition électronique

URL : https://journals.openedition.org/echogeo/13618

DOI : 10.4000/echogeo.13618

ISSN : 1963-1197

Éditeur

Pôle de recherche pour l'organisation et la diffusion de l'information géographique (CNRS UMR 8586)

Référence électronique

Frédéric Dejean, « De la religion dans les sciences sociales aux sciences sociales dans la religion : l'exemple chrétien », EchoGéo [En ligne], 26 | 2013, mis en ligne le 19 décembre 2013, consulté le 01 août 2021. URL : http://journals.openedition.org/echogeo/13618; DOI : https://doi.org/10.4000/ echogeo.13618

Ce document a été généré automatiquement le 1 août 2021.

EchoGéo est mis à disposition selon les termes de la licence Creative Commons Attribution - Pas d'Utilisation Commerciale - Pas de Modification 4.0 International (CC BY-NC-ND) 


\title{
De la religion dans les sciences sociales aux sciences sociales dans la religion : l'exemple chrétien
}

\author{
Frédéric Dejean
}

\section{Introduction}

1 Les travaux en sciences humaines et sociales traitant des faits religieux ont connu ces dernières années une forte croissance et reflètent ainsi la présence accrue de la question religieuse dans les débats publics, aussi bien en France que dans l'ensemble du monde occidental. L'anthropologue Clifford Geertz reconnait ainsi la pertinence de la religion comme objet de recherche et souligne combien «l'attention des sciences sociales s'était portée ailleurs, dominées qu'elles étaient par une série de présupposés évolutionnistes qui considèrent l'engagement religieux comme une force déclinante dans la société contemporaine » (Geertz, 2007, p. 427).

2 Cette croissance des productions scientifiques s'est accompagnée d'un intérêt accru de la société civile pour les travaux issus des sciences humaines et sociales. Des notions, concepts et théories ayant leur origine dans des travaux de recherche peuvent à l'occasion se diffuser hors des sphères académiques et se trouver mobilisés (Boltanski, 1990). Que l'on pense seulement à des concepts comme «habitus» ou «capital culturel » utilisés par des acteurs sociaux très divers, en dehors même de toute référence aux travaux de Pierre Bourdieu.

3 C'est donc dans ce double système d'intérêt - celui des sciences sociales pour les faits religieux et celui du grand public pour les productions scientifiques - que la question de la réception et des usages des recherches par les acteurs religieux doit être resituée.

Il apparaît ainsi que l'épistémologie des sciences sociales ne peut se limiter à la question de la production du savoir, mais doit également tenir compte du destin de ce savoir, une fois rendu disponible. De ce point de vue, l'essor des nouvelles technologies de l'information a joué un rôle déterminant dans la diffusion de travaux originaux en 
sciences sociales des religions, traditionnellement confinés à l'espace clos des bibliothèques universitaires. S'ils peuvent piquer la curiosité des groupes religieux dans la mesure où ils en sont les principaux protagonistes, il ne faut pas perdre de vue leur richesse pratique : en effet, plusieurs travaux - en particulier ceux qui portent sur les structures et sur les modalités de fonctionnement des communautés - constituent des invitations à une sorte d'auto-analyse institutionnelle.

5 Traitant de l'univers chrétien, nous proposons dans un premier temps de revenir sur la mise en place en France d'une sociologie religieuse et pastorale qui a précédé la formation de la sociologie des religions actuelle, et sur les dimensions géographiques qu'elle implique. Un tel exemple permet d'aborder des problématiques et des enjeux importants, et témoigne du fait que les usages des sciences sociales par les institutions religieuses sont intimement liés aux mutations urbaines. Dans un second temps, nous traiterons plus spécifiquement du lien entre les études urbaines et les usages que les catholiques français ont pu en faire. Enfin, dans un troisième temps nous introduirons l'entretien conduit avec Glenn Smith et indiquerons dans quelle mesure les communautés évangéliques s'adaptent aux mutations rapides des villes, en prenant appui sur des travaux de recherche venus des études urbaines.

\section{Avant la sociologie des religions, une sociologie pastorale}

6 En contexte français, la recherche portant sur les faits religieux ne s'est pas développée en opposition avec les institutions religieuses - principalement l'Église catholique mais au contraire dans un rapport étroit avec ces dernières, pour la simple raison que ces travaux avaient une visée pratique clairement affirmée et se trouvaient être l'œuvre de clercs ou de laïcs. Voici comment la revue Social Compass est présentée dans une note éditoriale de 1960 : «Le but de cette revue catholique consacrée aux études socioreligieuses est double: faciliter l'utilisation des méthodes sociologiques dans la pensée et l'action des chrétiens et apporter en sociologie la contribution des catholiques dans un domaine qui lui est propre, la sociologie de l'Église catholique " (cité par Mejido, 2004, p. 31). Dans ce cas de figure, les observateurs sont également des acteurs du milieu qu'ils étudient. Alors que cette position pourrait apparaître comme problématique, notamment pour la mise en œuvre du fameux devoir de neutralité axiologique, elle est au contraire présentée comme un atout : les catholiques seraient finalement les mieux placés pour parler du catholicisme.

$7 \mathrm{Au}$ sein de cette sociologie pastorale, les dimensions géographiques ne sont pas absentes puisque les nouvelles réalités spatiales du catholicisme doivent être comprises à des échelles multiples: que ce soit des analyses de la religiosité à l'échelle du territoire national ou bien des études plus fines concernant les pratiques dans les centres urbains ou dans les espaces ruraux. Dans un texte à valeur programmatique, Gabriel Le Bras - considéré comme un des pères de la sociologie des religions en France (Hervieu-Léger et Willaime, 2001) - met en avant la nécessité de constituer «des statistiques et des cartes expliquées, c'est à dire des tableaux précis, parlants, complets, de la pratique religieuse dans la France contemporaine " (Le Bras, 1931, p. 429). C'est dans un texte intitulé « Un programme : la géographie religieuse » (Le Bras, 1945) qu'il présente dans le détail les apports possibles de la géographie à la sociologie pastorale. Si la géographie doit être mobilisée c'est avant tout parce que le souci du sociologue est 
de quantifier les phénomènes dans l'espace, d'en étudier les répartitions, et d'établir des comparaisons. Le Bras écrit: "Chaque région, chaque terroir a sa topographie sacrée, sa nuance religieuse, parfois ses subdivisions infimes (...). Le programme de l'enquête devrait être établi successivement pour chaque unité, en partant de la plus élémentaire » (Le Bras, 1945, p. 88). Par la suite, ce relevé minutieux des faits religieux sur le territoire doit être traduit en un langage clair et synthétique; ce langage, est précisément celui de la carte. Le Bras va même jusqu'à apporter des éléments très pratiques: "Dimensions, couleurs et signes des cartes nouvelles seront choisis en accord avec les techniciens, quand ils auront mûri notre projet. L'échelle variera selon le paysage qui sera tantôt la France, tantôt un canton; nous choisirons des couleurs franches et bien distinctes, des signes simples et parlants » (Le Bras, 1945, p. 88-89).

\section{Les travaux sur la ville, un besoin d'expertise}

8 C'est en contexte urbain que l'usage des méthodes des sciences sociales par l'institution catholique est allé le plus loin. Plusieurs ouvrages concernent d'ailleurs directement les mutations des villes dans la période de l'après-guerre et des auteurs comme Gabriel Le Bras, Fernand Boulard (à la fois prêtre et sociologue) ou encore Jean Labbens (professeur aux Facultés catholiques de Lyon où il fonda l'Institut de Sociologie), interrogent les grands défis auxquels l'Église catholique doit faire face, notamment la profonde mutation socio-spatiale portée par l'affirmation d'une société urbaine. Alors que le modèle paroissial traditionnel coïncidait avec la civilisation rurale, l'urbanisation massive du territoire, l'essor des banlieues et plus largement la sécularisation de la société, constituent des ruptures majeures.

9 Non seulement les villes françaises sont les laboratoires de cette sociologie pastorale, mais surtout, ces types de recherches prennent à l'occasion appui sur des appareillages théoriques et méthodologiques particulièrement novateurs pour l'époque. Ainsi, avant même que les travaux de l'École de Chicago ne trouvent un large écho dans le milieu académique français, Jean Labbens (Chatelan, 2010) y puise des éléments de réflexion pour son ouvrage L'Église et les centres urbains (1958). Il se familiarisa avec les travaux de Burgess, de Park et de leurs collègues, à l'occasion d'un séjour aux États-Unis (1951-1952), mais également par la lecture attentive des travaux du sociologue et prêtre François Houtart qui séjourna et enseigna à Chicago. Dans son ouvrage Aspects sociologiques du catholicisme américain, paru en France en 1957, Houtart utilise le schéma demeuré fameux des zones concentriques en milieu urbain (Chatelan, 2010).

10 Si les chercheurs mobilisés sont majoritairement des sociologues, quelques géographes ont participé à l'occasion à ces travaux. Ce fut notamment le cas de Jean Labasse (Chatelan, 2009), bien connu aujourd'hui pour ses travaux novateurs en géographie régionale et en géographie économique. Dès le début de sa carrière, Labasse fut proche de l'association Économie et Humanisme, fondée en 1941 par un père dominicain. Au début des années 1960, les responsables catholiques lui confièrent la tâche de proposer un découpage du territoire français en grandes régions, dans le cadre d'une réflexion sur la réorganisation territoriale de l'Église catholique.

11 À Lyon, une vaste enquête sur les pratiques dominicales fut mise en place dans la première moitié des années 1950. Inscrite dans le sillage des travaux de Fernand Boulard et conduite par Jean Labbens, l'enquête avait pour principal objectif de « déterminer les obstacles de toutes natures qui entravent la pratique religieuse en 
ville » et de redéfinir le «maillage paroissial pour adapter le réseau des lieux de culte aux dynamiques de l'espace urbain" (Chatelan, 2012, p. 27). L'introduction de la sociologie dans le travail pastoral doit donc permettre d'établir un constat et de guider les prises de décision des responsables catholiques. Pour autant, un tel usage des méthodes issues des sciences sociales n'allait pas de soi, et des résistances s'exprimèrent. Pour un certain nombre de responsables religieux et de fidèles, pareille entreprise pouvait à terme "menacer le monopole de la théologie en matière de production de la vérité »(Chatelan, 2012, p. 27).

\section{Les études urbaines au service du travail missionnaire évangélique} chrétiens évangéliques et les rapports qu'ils entretiennent avec les travaux des sciences sociales. À l'instar de l'institution catholique dans le contexte lyonnais de l'aprèsguerre, consciente d'un nécessaire aggiornamento des structures paroissiales en milieu urbain, les évangéliques témoignent d'un effort constant d'adaptation de leurs structures institutionnelles et territoriales aux différents contextes dans lesquels ils évoluent. Cette attention aux effets de contexte est directement mise au service de l'entreprise missionnaire. Alors que l'institution catholique, fortement hiérarchisée, pouvait offrir certaines résistances aux changements de stratégie, l'univers protestant se caractérise par un éclatement institutionnel ayant pour conséquence une adaptation remarquable au contexte social et culturel.

13 L'observateur de la nébuleuse évangélique ne peut qu'être frappé par sa réactivité aux changements culturels de l'époque. Si le cœur du message ne varie pas, l'enveloppe évolue en fonction des sensibilités du moment, au point que la distance entre le caractère conservateur du discours et la modernité de la forme peut parfois étonner. Si les Églises développent des discours critiques face à l'esprit du temps et se présentent comme des espaces alternatifs, fonctionnant selon des normes clairement établies, non soumises au relativisme ambiant, il n'en demeure pas moins vrai qu'elles sont également particulièrement attentives à la culture dominante pour s'en réapproprier et en subvertir les codes. Ceci est particulièrement visible dans le domaine de la musique où il existe du rap chrétien, du heavy metal chrétien ou encore de la " pop louange » (Stowe, 2011).

qui donne lieu à une littérature importante, en particulier dans les milieux évangéliques nord-américains. Les évangéliques puisent principalement dans deux types de travaux : les recherches en géographie et en études urbaines qui traitent des mutations urbaines contemporaines, sans que l'accent ne soit mis sur les aspects strictement religieux, d'une part, et des travaux en sociologie des religions en contexte urbain, notamment les "congregation studies " (Ammerman, 1997; Ammerman et al, 1998 ; Chaves, 2004) et les études en " écologie religieuse " (Eiesland, 2000 ; Scheitle et Dougherty, 2008), d'autre part. Si les «congregation studies» s'intéressent spécifiquement au devenir des communautés religieuses dans le contexte contemporain, l'« écologie religieuse » situe ses travaux à un niveau plus directement spatial, puisqu'il s'agit de voir comment des communautés adaptent leur offre 
religieuse en fonction des mutations des espaces urbains dans lesquels elles se trouvent implantées.

Glenn Smith, au cours de l'entretien, explique comment les études urbaines et la géographie ont offert à des théologiens évangéliques des catégories et des notions dont ils ne disposaient pas. Les illustrations 1 et 2 montrent quelques livres de la bibliothèque de l'association montréalaise Direction Chrétienne dont Glenn Smith est le directeur. Des auteurs comme Richard Florida, Mike Davis ou encore Michael Dear côtoient des auteurs évangéliques.

Illustration 1 - Bibliothèque de Direction Chrétienne (détail)

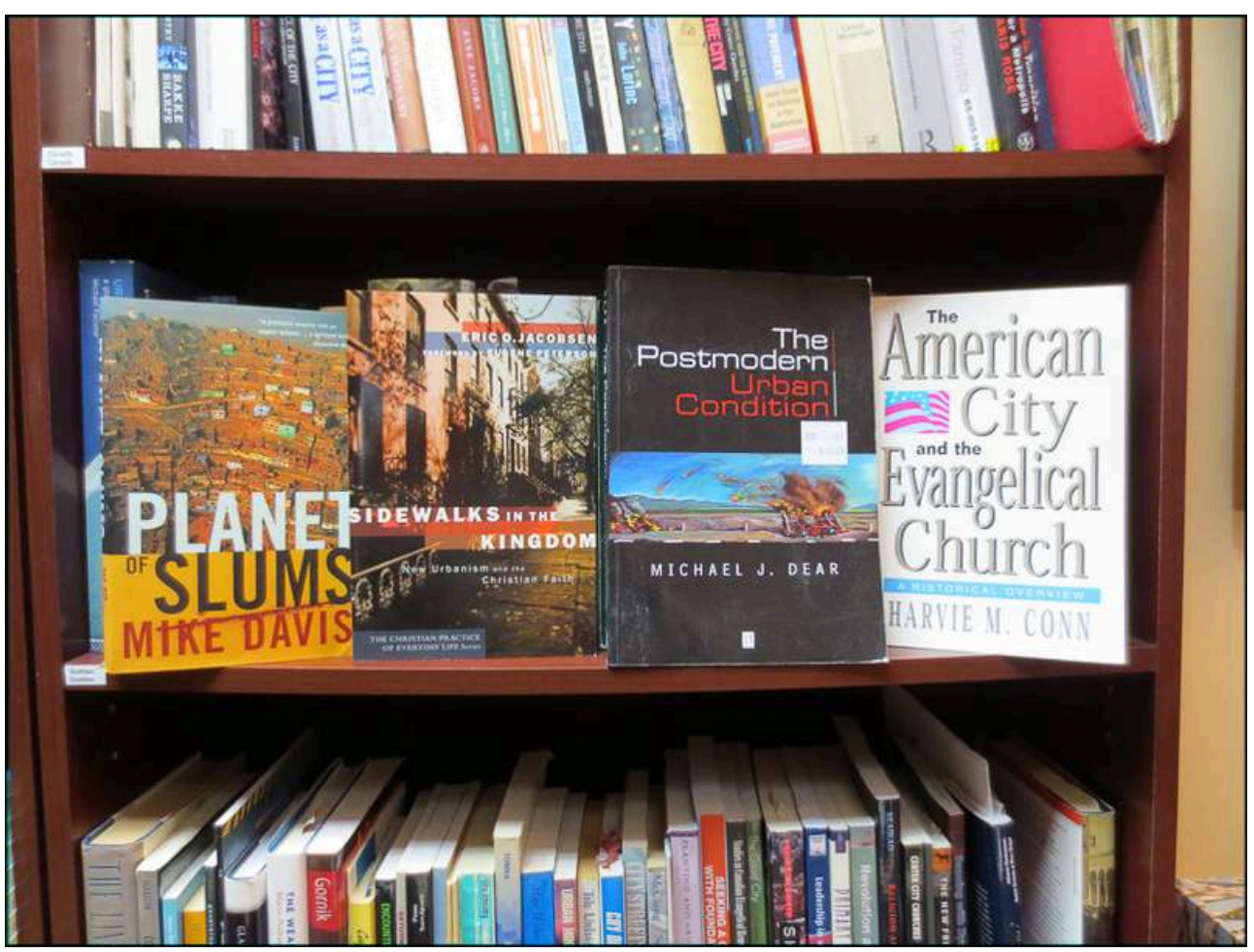

Auteur : F. Dejean, juin 2013, Montréal. 
Illustration 2 - Bibliothèque de Direction Chrétienne (détail)

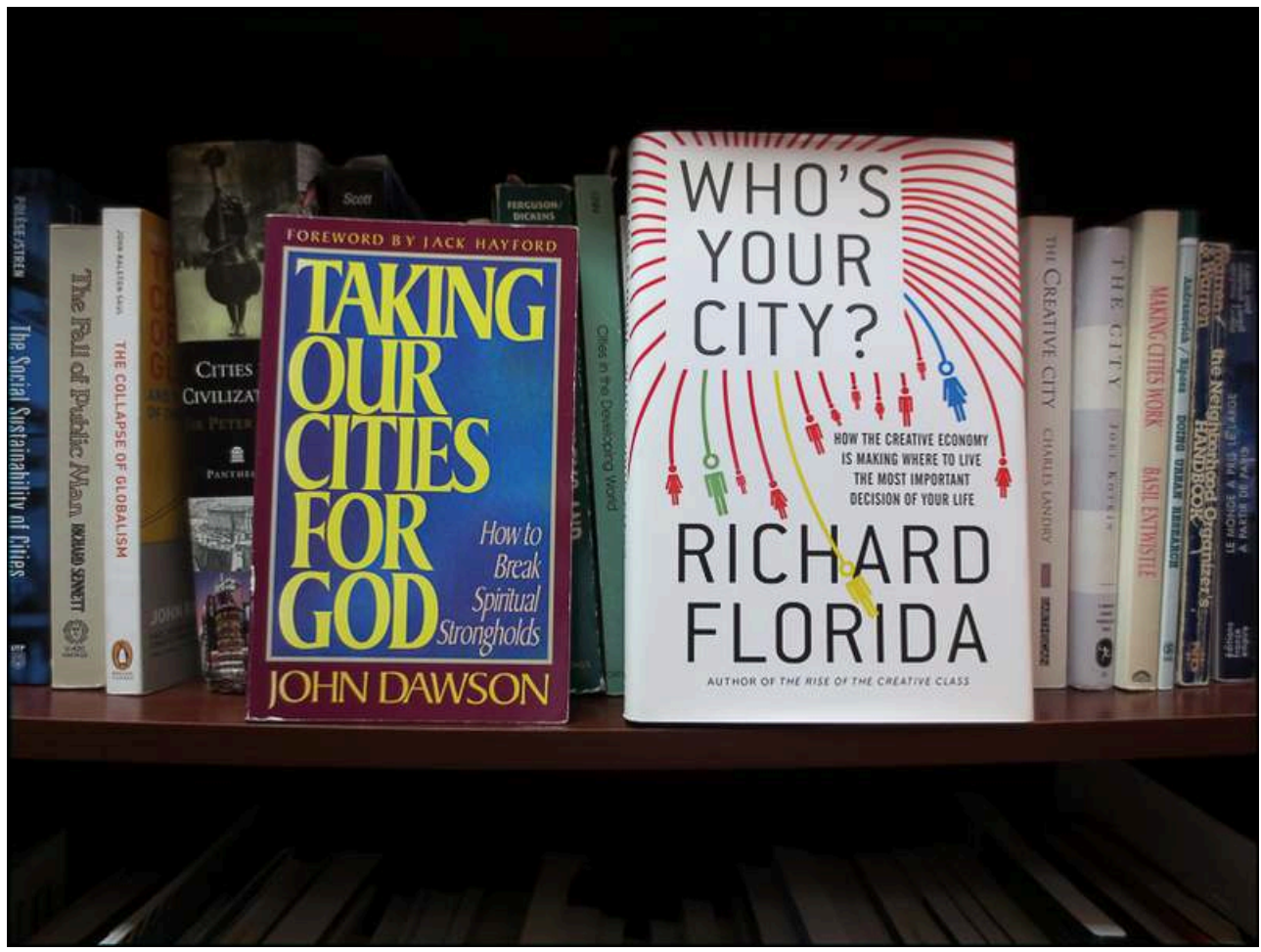

Auteur: F. Dejean, juin 2013, Montréal.

Comme dans le cas de la sociologie pastorale française, il faut distinguer l'intégration des recherches académiques dans la réflexion théologique de l'usage de méthodes scientifiques par des groupes religieux. Un des exemples les plus célèbres, d'ailleurs devenu un cas d'école dans les milieux évangéliques, est celui de Rick Warren, pasteur de la Saddleback Church, une des plus influentes megachurches américaines ${ }^{1}$. Avant d'ouvrir son Église, Warren a conduit une véritable «étude de marché » de façon à adapter l'offre religieuse à la demande et comprendre pourquoi des gens - pourtant croyants - ne fréquentaient plus les lieux de culte existants. Cette étude a pris la forme d'une enquête à partir de questionnaires, soumis à des résidents du comté d'Orange dans la banlieue de Los Angeles (Wilford, 2012).

\section{Entretien avec Glenn Smith}

17 L'entretien avec Glenn Smith illustre parfaitement la complexité des usages que les chrétiens évangéliques peuvent faire des études urbaines puisqu'elles se trouvent intégrées au cœur même du travail théologique et missionnaire. Glenn Smith est professeur de théologie pratique, spécialiste de la ville et de la mission urbaine. Chrétien anabaptiste ${ }^{2}$, il est le directeur de Direction Chrétienne, une organisation qui conseille les différentes dénominations chrétiennes dans leurs stratégies d'implantation et d'animation en milieu urbain. Tout au long de l'entretien ${ }^{3}$, Glenn Smith insiste sur le rôle des «théologies contextuelles » (Baum, 1990), expression qui recouvre un ensemble de courants théologiques rappelant que le message, discours religieux, ne peut se prévaloir d'une portée universelle, mais qu'il doit être constamment resitué dans un contexte spécifique. Dans le cadre d'une démarche en 
milieu urbain, les études urbaines offrent à la fois des catégories de pensée et des outils pratiques pour une telle théologie. L'entretien montre que les relations entre le discours scientifique et le discours religieux ne se nouent pas uniquement sur le mode de l'opposition, le premier étant du côté du savoir rationnellement fondé, tandis que le second serait du côté de la croyance, mais qu'il y a au contraire des points de passage et des zones d'interpénétration.

\section{Les sciences sociales dans l'appréhension des contextes}

- Frédéric Dejean (FD). Pouvez-vous présenter en quelques mots le parcours qui vous a conduit à développer une expertise pour les espaces urbains?

- Glenn Smith (GS). J'ai fait mes premières études en linguistique, en littérature française, et en sciences religieuses. J'ai finalement obtenu deux Bacs : un en science religieuse et l'autre en littérature française et en linguistique. Dans tous ces domaines, j'ai été conduit, même dans les années 1970, à interroger l'intégration de la culture dans la foi, le rôle de la culture et des différents enjeux que la culture soulève pour les croyants. Je me suis donc beaucoup intéressé à la sociologie et aux études culturelles. C'est quand j'ai fait ma maitrise en littérature patristique, celle des trois premiers siècles de l'Église, que j'ai pour ainsi dire découvert la ville. Le mouvement chrétien dans les premiers siècles était entièrement un mouvement urbain. J'ai étudié des personnes comme Irénée de Lyon. C'est vraiment en étudiant de près son ouvrage majeur, Contre les hérésies, que j'ai pris conscience qu'il s'agissait d'une réponse locale et contextualisée. D'autres auteurs des premiers siècles comme Saint Augustin ou Athanase insistent également sur le caractère urbain du christianisme.

À ce moment-là, j'ai débuté mes études de doctorat dans le champ de la «théologie contextuelle ", ce que l'on appelle maintenant la théologie à vocation missionnaire. Il fallait avoir un modèle de réflexion nouveau. Avec mes professeurs, nous avons développé une typologie qui ouvrait sur une fusion d'horizon entre le contexte et le texte. Par texte, dans la tradition protestante, on comprend les textes sacrés, les liturgies et en même temps l'histoire locale des communautés. J'ai donc dû développer une herméneutique pour lire le texte dans son contexte. Nous avons développé une façon de lire la ville de manière inductive qui s'accordait avec notre façon de lire les textes bibliques afin d'avoir une méthodologie cohérente. Mon mémoire de maitrise était sur cela. Évidemment depuis 1991, j'ai aiguisé ces réflexions.

21 Je me suis toujours demandé comment intégrer la foi et le vécu ecclésial dans un contexte social particulier. Dans les années 1980, quand nous avons commencé ces réflexions, on parlait alors beaucoup de culture, notamment avec la montée en puissance des études culturelles. Avec le processus de mondialisation, nous avons compris que la culture est devenue les cultures, surtout en milieu urbain. Il fallait donc avoir une nomenclature beaucoup plus appropriée afin de comprendre la localité. J'ai alors opté pour l'idée de la contextualisation qui met l'accent sur l'étude des contextes. Les études en géographie urbaine que j'ai lues, associées à la prise en compte de la pluralité des cultures dans les espaces urbains, ont été enrichies par la distinction entre le lieu et l'espace. Ce fut un vrai tournant. Par «lieu», j'ai compris le contexte géographique, le quartier, l'arrondissement, la ville. Et par "espace», j'ai compris l'imaginaire social. 

de la communauté ecclésiale, et également les effets de contexte. Ce faisant, la démarche devait impérativement être géographique et tenir compte de l'imaginaire social des résidents.

un premier temps, j'ai été fasciné par l'espace urbain québécois, surtout Montréal avec le système des deux rives, Nord et Sud. Il y a un phénomène urbain propre à Montréal, avec une riche histoire catholique et protestante. De fil en aiguille, je me suis mis à étudier les villes canadiennes et me suis alors rendu compte que très peu de personnes, à la fois praticiens et pratiquants, faisaient une théologie et une missiologie de la ville. Cela m'a ouvert beaucoup de liens et contacts. J'ai ainsi développé une expertise dans le champ des Églises dans le contexte des villes canadiennes.

Je continue d'étudier Montréal, surtout l'Église dans l'espace urbain, mais j'ai essayé ici de développer une certaine lecture des villes canadiennes car depuis trop longtemps, et cela persiste, les gens parlent de la ville nord-américaine alors même que c'est une catégorie un peu fourre-tout dans laquelle on veut faire tenir des réalités très différentes. Les villes de l'est et de l'ouest du Canada sont ainsi très différentes.

25 - FD. De ce que vous dites je retiens l'importance du thème de la culture avec l'émergence des études culturelles. On voit nettement ici une première influence dans le champ de la théologie par ce qui se fait dans les sciences sociales.

26 - GS. La grande nouveauté dans les années 1970 et 1980, c'était de lire les textes bibliques dans leur culture et dans leur contexte. Je peux vous montrer des volumes où les auteurs font un commentaire sur un texte biblique sans le resituer dans son contexte de production et de réception. Moi, j'enseigne à mes étudiants qu'il n'y a pas un livre dans le Nouveau Testament où l'auteur ne commence pas par préciser à qui il s'adresse. Ce ne sont pas des textes déculturalisés, décontextualisés. Il s'agit tout simplement de rappeler la structure sociale de la réalité d'un auteur à un moment donné, dans un contexte bien particulier. De ce point de vue, l'ouvrage de Peter Berger et Thomas Luckmann, La construction sociale de la réalité, m'a énormément influencé. Quand j'ai vu l'inculturation des textes bibliques j'ai vu la nécessité pour l'Église de se contextualiser.

27 - FD. La manière de poser les problèmes dans le champ de la théologie se trouve donc influencée par les travaux plus larges en sciences sociales?

28 - GS. Oui, définitivement.

\section{Similarités et différences des démarches}

29 - FD. Quelles seraient les différences entre un chercheur qui décrit les réalités urbaines canadiennes et vous qui décrivez la réalité des villes, car on peut finalement dire que vous faites des choses assez semblables?

30 - GS. La plus grande différence c'est que moi je suis un missiologue qui essaie d'articuler une missiologie et une théologie locale. Dans mon optique, Dieu s'est révélé dans la création, dans les textes sacrés, et aussi ultimement en Jésus Christ. La Bible est le grand récit, le métanarratif, du dévoilement de ce Dieu créateur. La théologie, c'est la réflexion humaine sur cette révélation à la lumière de nos propres circonstances. Cela signifie que n'importe quel théologien et missiologue porte des lunettes interprétatives. Et la théologie est un travail humain. Ceci étant dit, la Bible est 
vraiment une réflexion locale. Dans mon herméneutique, je vais toujours essayer de trouver le fil de l'unité dans le grand récit, de même que les particularités locales.

31 Une autre manière de faire la distinction est que nous allons toujours nous concentrer sur les particularités et les différences dans les villes canadiennes, plutôt que de parler des ressemblances ou des grandes caractéristiques. Pour nous, l'essentiel est de bâtir et de développer une Église ou un ouvrage missionnaire qui tient compte des particularités. Dans notre herméneutique urbaine, on enseigne aux étudiants à ne pas simplement faire des comparaisons mais à établir des contrastes. Je dis toujours aux étudiants que lorsqu'ils voient une différence de $5 \%$ par rapport à un trait social, ils doivent faire attention. Je trouve que trop souvent, la géographie urbaine - ou même les études culturelles - ne tiennent pas suffisamment compte des particularités et des contrastes. On veut toujours homogénéiser les résultats. Pour moi, c'est le contraire de ce Dieu de la création.

32 L'autre chose qui fait la différence est notre souci d'étudier l'imaginaire social des résidents et de comprendre comment ces imaginaires sont enracinés dans des lieux spécifiques. Il y a selon moi un chevauchement de l'imaginaire social et de la géographie locale.

33 - FD. Dans quelle mesure les travaux en géographie urbaine et en études urbaines ontils un impact sur la pratique de cette herméneutique urbaine?

34 - GS. Il y a toujours une présélection. Voici un exemple : dans le grand Montréal, il y a 864 secteurs de recensement pour les 3,8 millions de personnes. Dans 104 de ces 864 secteurs, le taux de pauvreté, les seuils de faible revenu ${ }^{4}$, dépasse $40 \%$. Quand j'étais enfant, à Toronto, j'aurais pu facilement vous conduire dans le quartier pauvre de Toronto. Quand je suis arrivé en 1975 à Montréal on pouvait facilement identifier le quartier dans lequel la pauvreté se concentrait. Aujourd'hui, ces 104 secteurs de recensements sont dispersés dans tout le territoire. Il n'y a pas une Église à Montréal qui peut poursuivre son action et sa réflexion sans tenir compte de cette dispersion géographique de la pauvreté. Cela a changé notre façon de penser.

35 C'est la même chose avec le phénomène du décrochage scolaire, un sujet qui nous touche particulièrement. Au Québec, plus de $50 \%$ des jeunes qui ont commencé le secondaire en septembre vont décrocher avant d'avant d'obtenir leur diplôme de fin d'études secondaires. Par ailleurs, un diplômé universitaire va commencer sa carrière avec un salaire annuel de $37000 \$$, alors qu'un jeune qui n'a jamais fini l'université va plafonner à $23000 \$$. Il y a donc ces phénomènes de disparités qui font qu'une Église ne peut pas fonctionner sans tenir compte de cette réalité.

36 - FD. Cela signifie que les travaux de recherche peuvent permettre de comprendre les dynamiques sociales et les effets de contexte. Comment vous positionnez-vous face aux dimensions politiques des travaux en géographie et en études urbaines?

37 - GS. Je vais répondre par un exemple. Dans le quartier de Hochelaga, un quartier très nationaliste et très canadien français, où nous sommes très engagés, nous observons une pauvreté qui est intergénérationnelle. Il y a 150 agences sociales financées par le fédéral, le provincial et le municipal, pour les 50000 personnes qui y habitent. La définition de la pauvreté dans ce quartier est uniquement économique. Notre lecture biblique nous démontre que la pauvreté n'est pas uniquement économique, mais également relationnelle. Et le pourcentage de personnes qui habitent seules est phénoménal. Cette attention aux personnes seules je le dois aux derniers travaux 
d'Annick Germain sur les "solos ». Aurons-nous une réponse purement économique? Notre lecture biblique nous pousse à tenir compte de la pauvreté spirituelle, physique et relationnelle. Il faut avoir une idéologie plus intégrale. Les gauchistes nous accusent d'être conservateurs et les conservateurs nous accusent d'être communistes. Je me dis que si je suis critiqué des deux côtés, c'est que j'ai sans doute raison.

Par ailleurs, nous ne craignons pas de parler d'une option préférentielle de Dieu pour les pauvres. Dieu a un souci envers les plus pauvres et les plus marginalisés car ils sont privés de la justice. Avoir des influences philosophiques et politiques, réfléchir dans des cadres, on l'admet parfaitement, et c'est d'ailleurs cela la construction sociale de la réalité. Mais nous reconnaissons qu'il faut être à l'écoute des textes bibliques.

Une des choses qui m'a vraiment aidé, c'est la prise de conscience de l'importance des "visions du monde » et la nécessité de toujours interroger les présupposés des acteurs. Je suis étonné que le Québec se trouve encore pris dans ce débat sur la laïcité et je rigole toujours un peu quand des fonctionnaires me parlent de la séparation de l'État et de l'Église. C'est une notion américaine et française. Il n'y avait jamais eu dans l'histoire du Canada une séparation entre les deux. Cela ne fait pas partie de notre histoire. Là on touche du doigt ce que peut être un présupposé. Il me semble préférable de développer une laïcité ouverte québécoise ou canadienne. Je ne veux pas que l'Église prenne le dessus dans la société, mais je ne veux pas non plus que l'État prétende qu'il est neutre.

\section{La pratique de l'exégèse urbaine et le rôle des études urbaines dans la compréhension d'un quartier}

40 - F.D. Pourriez-vous développer la notion d' " exégèse urbaine " qui est mise en avant à Direction Chrétienne, dans la mesure où cela ressemble par certains aspects au travail d'un chercheur?

41 - GS. Nous avons développé une méthodologie avec 20 étapes qui se présente comme un outil pratique mis au service des «implanteurs d'Église ». Au terme de ces étapes, on doit obtenir un portrait social, démographique, culturel, économique et politique du quartier dans lequel une Église souhaite s'implanter. Nous commençons avec les données de Statistiques Canada pour comprendre la composition sociale de la population. Par la suite nous proposons une méthodologie pour comprendre la place de l'Église dans sa localité, par rapport au pouvoir, aux médias, à sa propre histoire, aux institutions présentes.

42 Les analyses récentes sur Montréal laissent totalement de côté la place des Églises. Par exemple, dans Montréal: the quest for a metropolis rien n'est dit sur cette dimension. Avec la sécularisation, trop d'études urbaines négligent la place de l'Église dans les sociétés. Ce n'est pas un complot, mais c'est un aveuglement. J'apprécie le fait que, récemment, la géographie urbaine a redécouvert l'Église. Maintenant, il est certain que l'Église s'est marginalisée et elle a mérité sa marginalisation. Mais elle a redécouvert sa localité. C'est donc dans cette conjoncture que nous avons une richesse.

43 L'autre chose que les gens disent avec nos exégèses, c'est qu'elles sont lisibles. Trop souvent, les études urbaines sont trop théoriques et pas assez concrètes. Ce serait un peu comme une vulgarisation des travaux publiés par les chercheurs.

44 - FD. De ce fait, les études urbaines redécouvrent la religion, et la religion découvre ou redécouvre les travaux des sciences sociales... 
- GS. Oui, exactement. J'apprécie ce mouvement car il correspond exactement au cadre conceptuel que nous avons développé dans les années 1980 et qui prend vraiment racine dans une compréhension et une intégration des travaux venus des sciences. Je vais rencontrer dans quelques jours un jeune " planteur d'Église ». Il assiste l'Evangelical Pentecostal, une congrégation anglophone qui souhaite planter une église francophone. Ce jeune homme m'a donc écrit car avant de faire quoi que ce soit, il souhaite mieux comprendre la ville et ses différents quartiers. Il y a 30 ans, on n'aurait jamais eu une telle réflexion. L'Église découvre les sciences sociales.

\section{Les limites dans l'usage des sciences sociales}

46 - FD. Je crois qu'il y a un parallèle intéressant à faire avec la naissance de la sociologie des religions en France, qui fut d'abord une sociologie pastorale... Il y a parfois eu des réticences de la part des fidèles et des responsables religieux à introduire des méthodes des sciences sociales, car cela pouvait conduire à évacuer l'action divine au profit d'une rationalité strictement scientifique. Comment vous situez-vous par rapport à cela?

47 - GS. Je crois que le livre que vous m'aviez conseillé, Sacred Subdivisions, est excellent pour apporter un élément de réponse à cette question. J'ai trouvé que Justin Wilford ne tombait pas dans le réductionnisme. Il ne dit pas qu'il y a une explication uniquement banlieusarde dans la réussite de la Saddleback Church. Ça dépasse largement cela. Pour autant, il fait parfaitement ressortir le lien entre les megachurches et la suburbanisation aux États-Unis. Le chapitre sur les groupes de maison ${ }^{5}$ est tout à fait excellent et il montre bien comment ces petits groupes permettent de concilier le gigantisme et les rapports fraternels interindividuels.

48 Je trouve qu'il a pu expliquer certains phénomènes par la suburbanisation américaine. Le chapitre sur les petits groupes est absolument fascinant. Il a trouvé une niche pour Saddleback. Dans les cours de théologie pratique et d'études pastorales, je dis toujours aux étudiants de prendre les sciences sociales en général et les études urbaines en particulier pour ce qu'elles sont. Ne les forcez pas à faire quelque chose qu'elles ne sont pas compétentes à faire. De la même façon, ne demandez pas à la Bible de faire quelque chose qu'elle ne peut pas faire. Et pour cette raison, il y a un chevauchement, une complémentarité. Quand j'enseigne l'exégèse je commence toujours par Jonas à Ninive et Paul à Athènes, pour montrer que le texte biblique n'est pas silencieux pour l'intérêt des praticiens dans leur localité. Bien sûr, on ne peut pas étudier Montréal de la même façon que Jonas s'est intéressé à Ninive, mais nous avons des pistes de réflexion. Il s'agit donc de tenir compte des forces et des faiblesses, autant des textes bibliques que des théories urbaines.

\section{Succès évangélique et intégration de la culture}

49 - FD. De nombreux auteurs imputent le succès des évangéliques à leur capacité de réaction et d'adaptation à la culture environnante. De votre point de vue, est-ce que cela englobe la capacité à utiliser tous les moyens pour les mettre au service de l'évangélisation, même les travaux de recherche?

50 - GS. Un livre qui m'a énormément aidé est The Christian Mind: How should a Christian think de Harry Blamires. Dans cet ouvrage, il fait le plaidoyer d'une réflexion chrétienne sur tous les sujets. Quand j'ai lu ce livre pour la première fois j'ai pensé immédiatement 
qu'il fallait trouver une façon d'intégrer ma foi avec la réalité sociale. Je pense que les Évangéliques sont ceux qui vont le plus loin dans cette réflexion. C'est sans doute une force actuellement. Mais ne tombons pas dans la caricature, les Églises protestantes historiques et les Catholiques ont également fait ce travail, même s'ils sont aujourd'hui davantage dans un mode de survie. Par conséquent, la question n'est pas tant de savoir comment s'étendre que celle de savoir comment ne pas disparaître. Il est certain que l'étude d'un phénomène comme les megachurches montre à quel point les Évangéliques comprennent le branding et le marketing. Ils savent même où localiser leurs bâtiments par rapport aux systèmes de transport. De ce point de vue, ils ont une démarche quasiment scientifique dans la compréhension des réalités matérielles des congrégations.

51 - FD. Finalement, si l'on regarde le rapport que les chrétiens ont avec les études urbaines, nous voyons qu'elles permettent à la fois de poser des questions et d'offrir des méthodes. L'exemple de ce qu'avait fait Rick Warren est à ce titre éloquent?

52 - GS. Quand nous faisons les exégèses de quartiers, nous demandons toujours à ceux qui font les enquêtes sur le terrain de s'asseoir avec les gens et de discuter. Il est indispensable d'en passer par les fameux «stakeholder interviews ». On ne peut pas se contenter de faire des analyses démographiques et statistiques. Il est important de s'engager sur le terrain. Les auteurs de l'École de Chicago l'avaient déjà compris il y a près d'un siècle.

53 - FD. Les travaux des historiens, notamment ceux de Hugh McLeod, mettent en évidence les rapports ambigus que le christianisme a entretenus avec la ville. Aujourd'hui quels sont les défis de la ville contemporaine, et comment les travaux des chercheurs permettent-ils de réfléchir?

54 - GS. Sur le plan historique il est évident que l'époque médiévale fut un tournant dans l'urbanité de l'Église car tous les grands ordres étaient des mouvements ruraux. Finalement l'Église catholique romaine est retournée en ville. Le mouvement protestant dès le départ était rural et les grands mouvements missionnaires étaient carrément ruraux. Le mouvement protestant a peu d'histoire urbaine jusque vers les 1980. Les seules exceptions sont les Églises baptistes afro-américaines aux États-Unis. J'ai été chanceux d'avoir fait partie de cette première vague d'étudiants à explorer le mouvement urbain historique, de participer au travail d'appropriation de la ville.

Quand nous avons voulu nous réapproprier la ville, nous avons dû aller voir du côté des travaux des géographes de la ville, parce que la théologie ne disposait pas d'études et de méthodologie. Pendant le doctorat j'ai été conduit à prendre un cours de géographie urbaine. Même si j'étais en théologie je ne pouvais pas faire l'impasse sur la géographie. La géographie m'a définitivement aidé à penser de façon urbaine. Il y avait des catégories que je ne possédais pas avant. Ce fut une très belle découverte pour moi.

56 - FD. Si dans la ville du XIX ${ }^{e}$ siècle l'enjeu était la classe ouvrière, comme en témoigne un groupe comme l'Armée du Salut, quels seraient aujourd'hui les grands défis ?

57 - GS. L'initiative de l'Armée du Salut était excellente, mais c'était classiquement des activistes. Aujourd'hui, on sait l'importance de cette dialectique entre la réflexion et l'action. La géographie urbaine nous a aidés sur cette voie. Mais il a fallu faire dans un premier temps une réflexion théologique et prendre acte de l'urbanité de la Bible. Voici un exemple concret : il y a encore une vingtaine d'années un étudiant qui faisait sa maitrise en divinité dans certaines facultés, le seul cours pratique qu'il aurait suivi était 
un cours sur comment prêcher. Maintenant, dans nos programmes, un étudiant qui va terminer ses études du $2^{2 \mathrm{eme}}$ cycle est obligé de prendre un minimum de trois cours sur la ville. Un cours sur la théologie de la ville, la mondialisation et les grands enjeux et un $3^{\text {ème }}$ sur les études culturelles, la communication et la contextualisation. Nous avons maintenant une approche beaucoup plus pratique. Un jour, un étudiant m'a dit que nous lui avions enseigné la dogmatique, l'histoire de l'Église, l'exégèse des textes anciens, mais que personne ne lui avait enseigné comment négocier un bail ou un certificat d'occupation pour un lieu de culte. Il avait parfaitement raison. La formation était purement théologique : aucune utilité pratique.

- FD. Si l'on jette un coup d'œil à votre bibliothèque on peut observer des juxtapositions assez étonnantes : Planet of Slums de Mike Davis se trouve ainsi à côté de Taking our cities for God de John Dawson. Comment fait-on cohabiter ces auteurs?

59 - GS. Pour ma part, l'auteure qui m'a le plus influencé vient des études urbaines puisqu'il s'agit de Jane Jacobs dont j'ai lu tous les livres. Elle m'a donné des catégories locales et sa façon de penser en tenant compte des dimensions économique, sociale ou culturelle, m'a permis de m'approprier différents auteurs par la suite. Comme le rappelle Harry Blamires, il faut toujours sonder les présupposés des auteurs. Cela m’a aidé. Il ne s'agit donc pas de laisser de côté certains auteurs a priori, mais de les lire en tenant compte de leurs postulats de départ. Comme je suis avide de lectures nouvelles, j'essaie de ne pas simplement m'inscrire dans une philosophie, dans une école de pensée, mais d'avoir la vision la plus large possible de ce qui existe.

60 - FD. Est-ce que la fréquentation des travaux des sciences sociales ne conduit pas à rationaliser des choses qui resteraient sinon du domaine de l'inexplicable ou de l'ineffable? Un auteur comme Dawson propose une vision de la ville à proprement parler " enchantée », au sens wébérien. Cette tendance serait-elle comme un retour du balancier face à des approches trop marquées par les études urbaines?

61 - GS. Cela peut être une tendance. Par exemple quand j'enseigne à mes étudiants comment s'approprier les études culturelles et sociologiques pour la poursuite de la mission de Dieu, je les invite à éviter deux pôles : le réductionnisme qui permet de tout expliquer rationnellement et aussi un enchantement absolu où rien ne serait compréhensible en dehors d'une intervention divine. Je crois que nous devons tenir une position médiane entre ces deux pôles. C'est le va-et-vient entre ces deux tendances qui permet de produire un savoir utile. Encore une fois, on ne peut pas demander aux sciences sociales de faire ce qu'elles ne peuvent pas faire. Pour cette raison, dans notre herméneutique ecclésiale il y a l'étude des textes bibliques et l'étude des contextes. Il faut les contrebalancer sans cesse.

\section{BIBLIOGRAPHIE}

Ammerman N., 1997. Congregation and Community. Rutgers University Press, New Brunswick.

Ammerman N. (ed.), 1998. Studying Congregations: A New Handbook. Abingdon Press, Nashville. 
Baum G., 1990. La théologie contextuelle de Douglas Hall. Laval théologique et philosophique, 46(2). Bebbington D., 1989. Evangelicalism in Modern Britain: A history from the 1730s to the 1980s. Unwin Hyman, London.

Boltanski L., 1990. L'amour et la justice comme compétences. Métailié, Paris.

Boulard F., Rémy J., 1968. Pratique religieuse urbaine et régions culturelles. Éditions économie et humanisme/Les Éditions ouvrières, Paris.

Chatelan O., 2009. Les catholiques et la croissance urbaine dans l'agglomération lyonnaise pendant les Trente Glorieuses (1945-1975). Thèse de doctorat, Université Lumière Lyon 2.

Chatelan O., 2010. La migration comme modèle de compréhension de la ville dans l'expertise catholique (du début des années 1950 à la fin des années 1970). In Endelstein L., Fath S., Mathieu M. (Eds.), Dieu change en ville. Religion, espace, immigration. L'Harmattan, Paris.

Chatelan O., 2012. L'Église et la ville. Le diocèse de Lyon à l'épreuve de l'urbanisation (1954-1975). L'Harmattan, Paris.

Chaves M., 2004. Congregations in America. Harvard University Press, Cambridge MA.

Eiesland N. L., 2000. A Particular place: urban restructuring and religious ecology in a Southern suburb. Rutgers University Press, New Brunwick.

Geertz C., 2007. La religion, sujet d'avenir. In Wieviorka M. (ed.), Les sciences sociales en mutation. Éditions Sciences humaines, Paris.

Hervieu-Léger D., Willaime J.-P., 2001. Sociologie et religions, approches classiques. Presses Universitaires de France, Paris.

Labbens J., 1958. L'Église et les centres urbains. Spes, Paris.

Le Bras G., 1931. Statistique et histoire religieuses. Revue d'histoire de l'Église de France, 17(77).

Le Bras G., 1945. Un programme: la géographie religieuse. Annales d'histoire sociale, 8(1).

Mary A., 2006. Les Archives... Cinquante ans... après. Archives des Sciences Sociales des Religions, (136).

Mejido M. J., 2004. On the Genesis and Transformations of Social Compass. Social Compass, 51(1).

Scheitle C., Dougherty K., 2008. The Sociology of Religious Organizations. Sociology Compass, (2/3).

Stowe D., 2011. No Sympathy for the Devil. Christian pop music and the transformation of American Evangelicalism. University of North Carolina Press, Chapel Hill.

Wilford J., 2012. Sacred subdivisions. The postsuburban transformation of American Evangelicalism. New-York University Press, New-York.

\section{NOTES}

1. Pour une présentation de la Saddle Back Church, voir http://geographie-religions.com/ 2013/04/29/une-visite-a-la-saddleback-church. On appelle communément megachurch une assemblée dont le nombre de fidèles excède les 2000 personnes. On parle parfois de gigachurch quand la barre des 10000 fidèles est franchie.

2. Apparu dans la première moitié $d u X V I^{e}$ siècle dans ce qui est aujourd'hui l'Allemagne, l'anabaptisme se caractérise par le refus du baptême des nouveau-nés, allant ainsi à rebours de la pratique catholique traditionnelle. 
3. Cet entretien s'est déroulé en juin 2013 dans les locaux montréalais de Direction chrétienne.

4. «Les seuils de faible revenu (SFR) visent à mesurer le niveau de revenu pour lequel une famille peut se trouver dans des circonstances difficiles du fait qu'elle doit dépenser une proportion plus grande de son revenu sur des articles de base (alimentation, logement et vêtements), comparativement à une famille moyenne de même taille. Les SFR varient en fonction de la taille de la famille et de la taille de la région de résidence ». Source : Statistiques Canada, http:// www5.statcan.gc.ca/bsolc/olc-cel/olc-cel?catno=13-551-XIB\&lang=fra

5. Au chapitre 5 ( Purpose Driven Places Small Performances in Big Churches ») de son ouvrage, Justin Wilford explique comment l'intégration à la Saddle Back passe davantage par une participation active à des groupes de maison d'une douzaine de personnes habitant dans le même quartier, que par la présence hebdomadaire sur le campus de l'Église

\section{AUTEUR}

\section{FRÉDÉRIC DEJEAN}

Frédéric Dejean, frederic.dejean@yahoo.fr, titulaire d'un PhD Études urbaines est Chercheur post-doctoral à l'Institut d'Urbanisme, Université de Montréal. Il a publié récemment :

- Dejean F., Endelstein L, 2013. Approches spatiales des faits religieux. Jalons épistémologiques et orientations contemporaines. Carnets de Géographes, $\mathrm{n}^{\circ} 6$, http://www.carnetsdegeographes.org/ carnets_debats/debat_06_01_Dejean_Endelstein.php.

- Dejean F., Koussens D., 2013. Stratégies des communautés évangéliques face aux contraintes des règlementations des lieux de cultes en France et au Québec. Studies in Religion/Sciences religieuses, Vol. 42, No 1, p. 59-82.

- Dejean F., 2012. Pierre Deffontaines, géographe de la « Noosphère ». Une lecture de Géographie et religions. Cahiers de Géographie du Québec, Vol. 56, N 159, p. 543-556. 